\title{
CLINICAL PRESENTATION AND MANAGEMENT OF AMOEBIC LIVER ABSCESS
}

\author{
Naveen $S^{1}$, Hariprasad T. R², M. Vishnu Vardhan Reddy³, Rohit Krishnappa ${ }^{4}$ \\ 1 Professor, Department of General Surgery, Sree Rajarajeshwari Medical College, Bangalore. \\ ${ }^{2}$ Associate Professor, Department of General Surgery, Sree Rajarajeshwari Medical College, Bangalore. \\ ${ }^{3}$ Resident, Department of General Surgery, Sree Rajarajeshwari Medical College, Bangalore. \\ ${ }^{4}$ Assistant Professor, Department of General Surgery, Sree Rajarajeshwari Medical College, Bangalore.
}

\section{ABSTRACT}

\section{BACKGROUND AND OBJECTIVES}

ALA has gained importance during recent years as a result of increasing world travel, economic globalization and the growing number of chronically immunosuppressed people. AIDS and the increasing use of organ transplants have led to a new population at risk. In our study we aimed to analyse the clinical presentation and management of Amoebic liver abscess at Sree Rajarajeshwari Medical College and Hospital, Bangalore.

\section{METHODS}

A hospital based prospective observational study was conducted between Dec. 2013 to June 2015 in Sree Rajarajeshwari Medical College and Hospital, Bangalore. All patients admitted with suspicion of liver abscess were confirmed with ultrasonography. After establishing sonological diagnosis according to criteria, the treatment was started from the day of admission which consisted of Antibiotics, USG guided Aspiration, Pigtail catheterization and Open surgical Drainage.

\section{RESULTS}

Forty five cases of Amoebic liver abscess were studied; 28 patients (62\%) were between 21 and 40 years of age. Male-to-Female ratio was 14:1. All the patients were from rural background. History of alcoholism was present in 10 patients $(22.2 \%)$.

Plain X-ray abdomen showed signs of peritonitis in 2 patients.

Right lobe of liver was involved in 40 cases and left lobe in 3 cases. Both lobes were involved in 2 cases.

Twenty six cases were treated conservatively, 11 cases by aspiration, 6 patients by pigtail catheterization.

Two patients underwent open surgical drainage for perforated liver abscess.

Complications noted in our series were rupture into peritoneal cavity in 2 cases; mortality was seen in 1 case.

\section{CONCLUSION}

Amoebic liver abscess is still one of the commonest parasitic infections of the liver in developing countries. Prompt diagnosis, aggressive medical treatment along with minimal intervention can keep the morbidity and mortality associated with this condition to a bare minimum. The scope of surgery in this condition is minimal and limited to complications of rupture.

\section{KEYWORDS}

Amoebic Liver Abscess; Image Guided Drainage; Peritonitis; Surgical Drainage.

HOW TO CITE THIS ARTICLE: Naveen S, Hariprasad TR, Reddy MVV, et al. "Clinical presentation and management of amoebic liver abscess". Journal of Evolution of Medical and Dental Sciences 2015; Vol. 4, Issue 104, December 28; Page: 16915-16918,

DOI: $10.14260 /$ jemds/2015/2547

\section{INTRODUCTION}

Abscess formation within the liver occurs in a variety of circumstance and in response to different agents. Abscess of the liver may be pyogenic or parasitic in origin. With introduction of antibiotics, the incidence of pyogenic abscess of the liver has decreased to greater extent. ${ }^{1}$

Liver abscess is the most common extra-intestinal manifestation of amoebiasis. Hepatic amoebiasis is reported in $3 \%$ to $10 \%$ of afflicted patients. The incidence is high in tropical countries and it is attributed to lack of proper sanitation and personal hygiene due to low socio-economic conditions. ${ }^{1}$ The disease is prevalent world over. In Indians it affects especially people from Northeastern regions of the country.

\section{Financial or Other, Competing Interest: None.}

Submission 03-12-2015, Peer Review 04-12-2015,

Acceptance 22-12-2015, Published 24-12-2015.

Corresponding Author:

Dr. Rohit Krishnappa,

Assistant Professor

Department of General Surgery,

Sree Rajarajeshwari Medical College,

Kambipura, Bangalore-560074.

E-mail: rohitkrishnappa@yahoo.co.in

DOI: $10.14260 /$ jemds/2015/2547
It is clearly a public health problem of international importance.

Several factors such as different strains of E. Histolytica, patients' susceptibility, alcoholism and malnutrition predispose to the disease.

After innovation of imaging techniques such as ultrasound, isotope scan, CT scan, serological tests. The diagnosis of liver abscess can be made early, rapidly and accurately. ${ }^{2}$

It is imperative to distinguish between the amoebic and pyogenic types of hepatic abscess because diagnosis, treatment and prognosis differ in many respects.

The morbidity and mortality of amoebic liver abscess should approach zero with proper use of diagnostic and minimally invasive therapeutic measures available now.

\section{MATERIALS AND METHODS}

A hospital based prospective observational study was carried out in the surgical wards of Rajarajeshwari Medical College and Hospital, Bangalore and the initial diagnosis of amoebic abscess was made depending on the typical features on USG.

Detailed history of all patients was taken with thorough clinical examination and entered into proforma during their stay and followup. 
Response to Anti-amoebic drugs was studied. Demonstration of characteristic pus (Anchovy sauce) on aspiration confirmed the diagnosis.

\author{
The following investigations were carried out for all \\ patients \\ - $\quad$ CBC with ESR, LFT, Urine routine. \\ - Coagulation profile. \\ - $\quad$ Chest X-ray, PA view. \\ - Plain X-ray, Abdomen. \\ - Stool examination for Entamoeba Cyst/Trophozoites. \\ - Ultrasonogram of abdomen. \\ - Culture of the aspirate/abscess material.
}

After establishing clinical diagnosis the treatment was instituted from the day of admission, which consisted of:

Oral or intravenous metronidazole in a dose of $400 \mathrm{mg}$ three times a day.

Supportive therapy was given in the form of intravenous fluids, analgesics and protein rich diet. Seeing response to this therapy patient were selected for aspiration or open surgical drainage.

The response to therapy was assessed with particular regard to subjective symptoms and signs like fever, hepatic size, tenderness and laboratory and radiological findings.

The patients were kept in hospital during the entire course of therapy.

Descriptive statistics were used to express the data.

Data were presented in tabular and graphical forms.

\section{RESULTS}

A $0.5 \%$ of total surgical admissions were cases of Amoebic Liver Abscess.

Amoebic liver abscess was more common in the age group between 21 and 40 years, who constituted $62 \%$ of total no. of cases in the present study. Youngest patient encountered was 22 years and oldest was 65 years (Table 1 ).

Out of 45 cases, 42 were male (93\%) and 3 were female (6.6\%) (Table 2). Ratio of male-to-female was 14:1. Three cases $(6.6 \%)$ gave past history of dysentery and no one presented with symptoms during the study. The duration of symptoms varied from 1 day to 1 year; 43 patients presented with acute onset of symptoms and the remaining 2 were chronic cases. With regard to symptoms in these 45 cases studied, it was observed that pain $(n=26,57.7 \%)$ in the right upper quadrant and pain in the upper abdomen dominated the clinical picture (Chart 1).

The next commonest symptoms were nausea and vomiting in 16 patients (35.5\%) and jaundice in 5 patients (11.1\%) (Chart 2). Other associated symptoms were anorexia, loss of weight and lassitude.

Palpable enlarged liver was encountered in 18 cases (40\%). Intercostal tenderness was present in 26 cases (57\%). Tenderness all over abdomen was present in 2 cases (4.4\%). Patients who presented with septic and shock was 2 (4.4\%). Anaemia was seen in 8 cases (17.7\%) (Patients with less than $10 \mathrm{mg} / \mathrm{dl}$ were considered anaemic) (Chart 3 ).

Leukocyte counts more than 10,000 cells/cu mm was found in 6 patients (13.3\%). ESR was raised above $20 \mathrm{~mm} / \mathrm{hr}$ in 25 patients $(55.5 \%)$. Elevated levels of Serum Billirubin $(>2 \mathrm{mg} \%)$ in 5 patients $(11.1 \%)$ in the present series. Serum transaminases were raised in 20 patients (44.4\%).

Erect X-ray abdomen showed signs of peritonitis in 2 patients (4.4\%). On Chest X-ray, 42 patients had restricted movement of the effected side of diaphragm and also raised hemidiaphragm. Ultrasonogram of abdomen was done in all patients with suspicion, which showed evidence of amoebic liver abscess. Right lobe involvement was present in 40 cases $(88.6 \%)$ and left lobe in 3 cases $(6.6 \%)$ and both lobes involvement in 2 cases (4.4\%). Right lobe of the liver was more often involved than the left lobe (Table 4). Solitary abscess was noted in 43 cases $(95.6 \%)$ and multiple abscesses in 2 cases (4.4\%) (Table 3).

All patients were started on medical line of management, i.e. in 43 patients. Out of these, 26 patients $(57.7 \%)$ responded to this treatment alone.

Aspiration was done in 11 patients with a volume of the abscess in range of $100 \mathrm{ml}$ to $200 \mathrm{ml}$. Patients were evaluated for clinical improvement between 48-72hrs; they were monitored for complications like rupture and for signs of secondary infection. In 4 cases aspiration was repeated twice. All 11 patients responded to aspiration alone and there was no further intervention done.

Pigtail catheterization was done in 6 patients in whom the volume was $>200 \mathrm{ml}$.

Open surgical procedure was done in 2 patients who presented with rupture. A patient with ruptured liver abscess expired (Chart 4).

\begin{tabular}{|c|c|c|}
\hline Age Group & No. of Cases & Percentage \\
\hline $0-10$ & 0 & $0 \%$ \\
$11-20$ & 0 & $0 \%$ \\
$21-30$ & 14 & $31.1 \%$ \\
$31-40$ & 14 & $31.1 \%$ \\
$41-50$ & 10 & $22.2 \%$ \\
$51-60$ & 5 & $11.1 \%$ \\
61 and above & 2 & $4.4 \%$ \\
\hline Total & 45 & $100 \%$ \\
\hline
\end{tabular}

Table 1: Age Distribution

\begin{tabular}{|l|c|c|}
\hline Sex & No. of cases & Percentage \\
\hline Male & 42 & $93.3 \%$ \\
Female & 3 & $6.6 \%$ \\
\hline
\end{tabular}

Table 2: Sex Distribution

\begin{tabular}{|c|c|c|}
\hline No. of Abscesses & No. of Cases & Percentage \\
\hline Solitary & 43 & $95.6 \%$ \\
\hline Multiple & 02 & $4.4 \%$ \\
\hline
\end{tabular}

Table 3: Lesions

\begin{tabular}{|l|c|c|}
\hline Location & No. of Cases & Percentage \\
\hline Right lobe & 40 & $88.8 \%$ \\
Left lobe & 03 & $6.6 \%$ \\
Both lobe & 02 & $4.4 \%$ \\
\hline
\end{tabular}

Table 4: Site of Lesion 


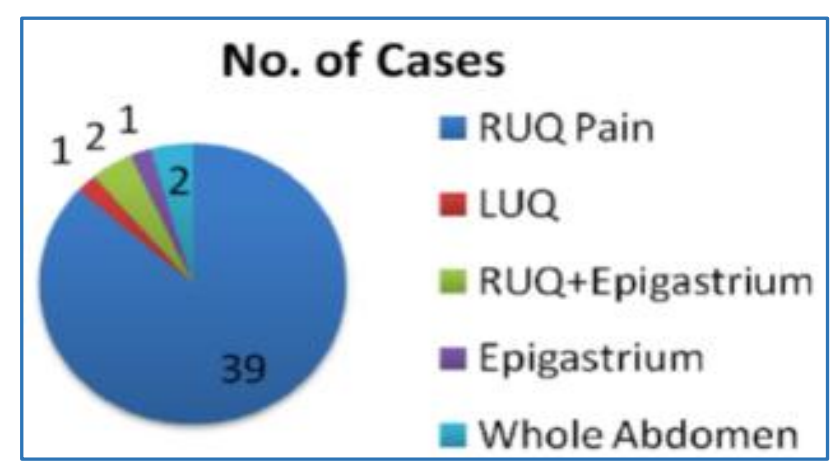

Chart 1: Symptom of Pain

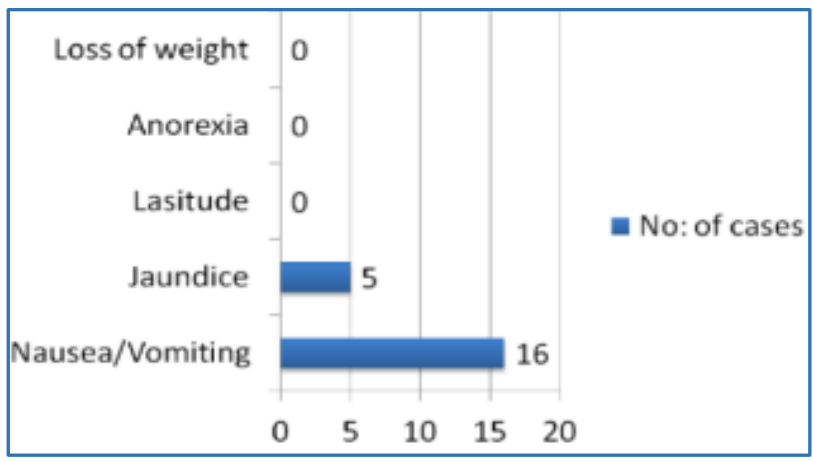

Chart 2: Other Symptoms

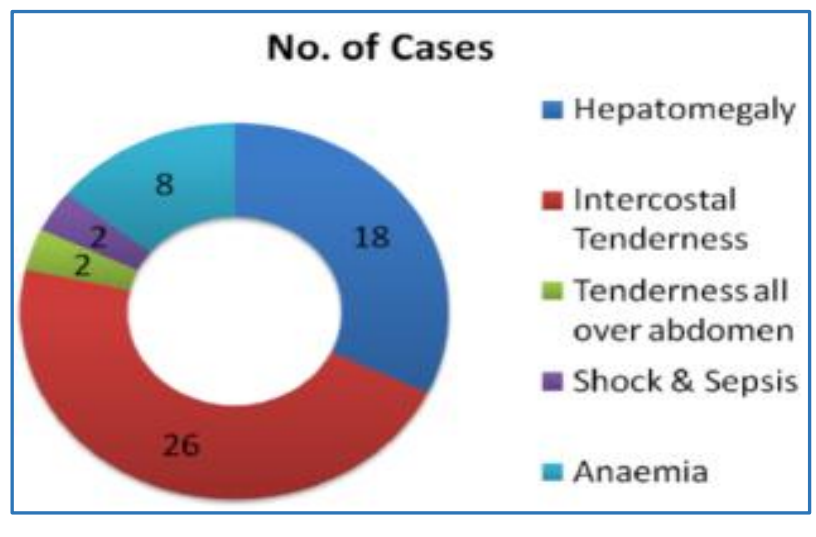

Chart 3: Signs

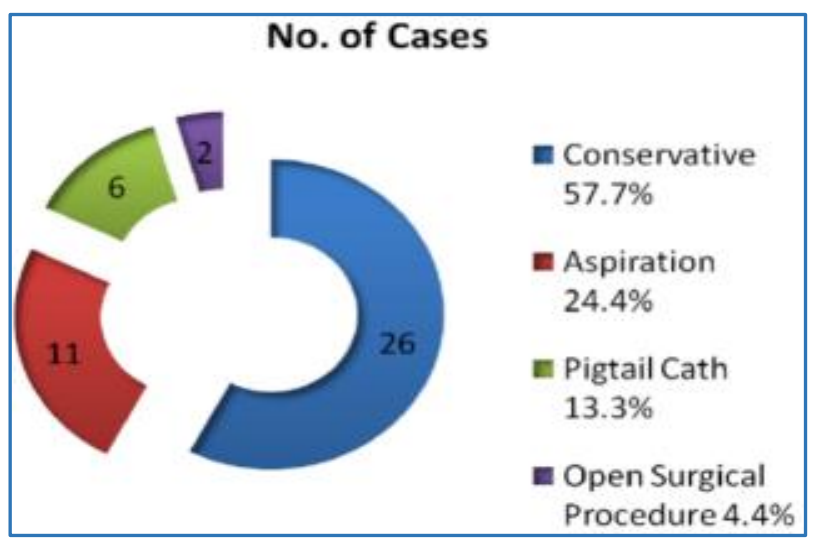

Chart 4: Management

\section{DISCUSSION}

Amoebic liver abscess is the one of the most important and frequent complications of amoebiasis. The incidence is high in tropical countries and it is attributed to lack of the proper sanitation and personal hygiene due to low socio-economic conditions.
Despite advances in the treatment, amoebic liver abscess is still the commonest and the most serious complication of amoebiasis. This is one of the illness to which there are a variety of effective drugs and surgical help is available once it is diagnosed. Hence, early diagnosis and treatment of the illness can prevent the complications and sequelae.

In the present study, the common age group found was between 21 and 40 years and it constituted $62 \%$ of the total cases. Similar results have been reported by Mishra et al. and Singh and Srivastava studies. ${ }^{3}$

Males (93.3\%) were predominantly affected with amoebic liver abscess in the present study, which were similar to other studies like Debakey and Oschner, Raghavan et al., Habibullah et al. and Katzenstein et al.4,5,6,7

Out of 45 cases, there were 42 male patients (93.3\%) and 3 female patients (6.6\%). The male-to-female ratio is $14: 1$. Any satisfactory explanation is yet to come forth as to why males are more frequently affected than females. It can be explained because of alcoholic habits in males. In present study, 10 patients $(22.2 \%)$ were alcoholic.

Iron is essential for the growth of E. histolytica and lack of it keeps the parasite weak and docile. Perhaps the lower incidence of amoebiasis in women is from their iron deficiency state, but their observation needs further study.

All the patients in the study were from lower socioeconomic status. It is well known that amoebiasis is common in slum dwellers due to bad sanitation, poverty, ignorance and their poor nutritional status.

In the present study 3 cases (6.6\%) gave past history of dysentery and no one presented with symptoms during the study period. Raghavan et al. recorded only $9.8 \%$ of the 193 cases with dysenteric symptoms. Barbour and Janiper in their study of 33 cases could record a history of accompanying dysentery in $55 \%$ of cases. ${ }^{8}$ Nanda and Tandon noted past history of dysentery in $25 \%$ of their 80 patients. ${ }^{9}$ Carneiro Chaves et al. reported $22 \%$ of their cases with past history of dysentery. ${ }^{10}$

Pain was a complaint in all the cases in this study. It was present in upper abdomen. The nature of the pain was dull aching in majority of the patients and it was of a sharp stabbing nature in a few. The incidence of pain in the present study is similar to the studies done by Poulose et al. Nanda and Tandon. ${ }^{11,9}$ Fever was present in 26 cases (57\%). The commonest pattern of fever noted in our series was low grade. After the initiation of the therapy, fever was one the earliest symptom to subside.

A palpable and enlarged liver was encountered in 18 cases (40\%). It is one of the most essential parameters and a consistent sign in amoebic liver abscess. The liver is usually tender. Different workers have reported Hepatomegaly from $60-100 \%$ in the cases studied. Debakey and Oschner noted in $77.20 \%$ cases. Mehta and Vakil noted in 98\% cases. ${ }^{12}$ Katzenstein et al. suggests that Hepatomegaly is more common in chronic cases.

Intercostal tenderness was one of the diagnostic signs of amoebic liver abscess, but absence of it does not rule out the diagnosis. It was noted in 26 cases $(57.7 \%)$ in present series. Mehta and Vakil reported in $19 \%$ of their cases, whereas Bardour and Juniper found in $71.2 \%$ of their cases.

Jaundice was noted in 5 cases $(11.1 \%)$ in the present study. In all the cases, jaundice improved by 1 to 2 weeks after initiation of therapy. Joshi et al. and Mansons-Bahr reported similar results. ${ }^{13,14}$

Jaundice is more common in cases with left lobe abscess. The mechanism of jaundice is partly due to periductal inflammation and pressure by a large abscess on the bile duct. Manifestations of pleuro-pulmonary involvement on right side are common. But in our study, no patients presented with lung signs. 
Raghavan et al. reported pleural effusion in 19 cases (15.5\%) and consolidation of right lung base in 45 of 126 cases studied.

Anaemia was one of the common finding, accompanying amoebic liver abscess. Anaemia is very common in chronic cases and is due to chronic infection. Adams and Macleod found anaemia in $63 \%$ of their cases studied. ${ }^{15}$ They studied serum iron and bone marrow and found it to be normal and concluded anaemia to be due to the presence of chronic infection.

Leukocyte counts more than 10,000 cells was found in 6 patients $(13.3 \%)$. This is one of the parameters in assessing the prognosis after initiation of therapy. Various workers have reported leucocytosis in amoebic liver abscess as follows:

Adams and Macleod reported $77 \%$ of their cases had leukocytosis. Katzenstein found 63 cases in their 67 cases had the rise. Usually, polymorphonuclear leucocytes predominate. Mansor Bahr and Willough referred to the lack of elevation leukocyte count being feature of amoebic liver abscess; however, the marked elevation of leukocyte count helps to distinguish between pyogenic liver abscess and amoebic abscess.

The correlation between degree of anaemia, leucocytosis and duration of illness reveal that patients with a short history tend to show no anaemia, but appreciable leucocytosis, whereas those with a long history tend to show appreciable anaemia with less marked leucocytosis. In the present series high leukocyte counts were noted in patients with acute history and also were associated with moderate anaemia.

A raised ESR is almost a constant finding in amoebic liver abscess. In the present study ESR was raised above $20 \mathrm{~mm} / \mathrm{hr}$ in 25 patients $(55.5 \%)$. It is one of the essential parameter/criteria in assessing the response of drug therapy.

The value of liver function tests in liver abscess has been a subject of dispute. These tests may not be absolutely diagnostic, but are helpful in assessing the outcome of the disease. However, some recent papers have emphasized the value of liver function tests diagnosis of liver abscess.

Serum bilirubin was raised $(>2 \mathrm{mg} / \mathrm{dl})$ in 5 cases $(11.1 \%)$ in the present study. Clinically, jaundice was detected in 8 cases. It was mild and hepatocellular in type. The incidence of jaundice reported by several workers varies from $10-50 \%$. Serum transaminases was raised in 8 cases $(17.7 \%)$ in this series. SGOT was raised in $50 \%$ of cases in Katzenstein et al. series and in 33 cases (41.2\%) of Nanda and Tandon series. In the same series, SGPT was raised in 29 cases (36.2\%).

For Serum Alkaline Phosphatase a value of above $13 \mathrm{KA}$ units was considered abnormal. It was raised in 8 cases $(17.7 \%)$ in the present series. Abnormally high alkaline phosphatase levels were the most reliable biochemical indicators of amoebic liver abscess. It was evaluated in $84 \%$ of cases of Katzenstein et al. According to them higher values were found in chronic amoebic liver abscess. Persistent high alkaline phosphatase levels suggest non-responsiveness of the abscess to drug therapy and it is an indicator for needle aspiration of the abscess.

It is not possible in every case to demonstrate trophozoites or cysts of E. histolytica in feces examination or from the material obtained on procto-sigmoidoscopic examination. Trophozoites or cysts are demonstrable in the feces only in about one third of the patients with abscesses.

Out of 45 cases in the present study, one case $(2.2 \%)$ showed E. histolytical cysts in stool. Debakey and Oschner found E. histolytica trophozoites in $15.4 \%$ of cases. Yadav et al. found in 20\% of cases, whereas Carneriro Chaves found trophozoites in $7.8 \%$ of their cases.

Fluoroscopy is undoubtedly one of the best and most significant diagnostic measures in amoebic liver abscess.
Elevation of the hemidiaphragm with diminished movements was found in 43 patients (95.5\%) in this present series.

Various workers have reported similar findings. Singh and Srivastava found $84 \%$ having positive results. Habibulla et al. in $60 \%$ of cases and Carneiro Chaves found positive results in $67 \%$ of their cases.

Percutaneous needle aspiration was done in 11 patients $(24.4 \%)$ and percutaneous pigtail catheterization was done in 6 patients $(13.3 \%)$.

Manson-Bahr expresses that the mere presence of anchovy sauce signifies positive diagnosis of amoebic liver abscess.

If the abscess cavity is connected with the biliary tree then the aspirate is greenish. Kapoor has found various colors other than chocolate color in his cases. In present study, majority of the patients had chocolate colored pus.

\section{CONCLUSION}

From our experience of a small study group, we conclude that amoebic liver abscess is still one of the commonest parasitic infections of the liver in developing countries. The clinical presentation is universally almost same. Prompt diagnosis, aggressive medical treatment along with minimal intervention can keep the morbidity and mortality associated with this condition to a bare minimum. With the availability of modern imaging techniques and minimal access modalities better results can be achieved. The scope of surgery in this condition is minimal and limited to cases with complications of rupture.

\section{REFERENCES}

1. Wintch RW, Reines HD, Rambo WM. Liver abscess: A changing entity. Am Surg 1982;48:11-5.

2. Jorden PH, Jr. Treatment of amoebic abscess of liver by open surgical drainage. Ann surg 1955;141:70.

3. Singh VP, Srivastava SN, Srivastava PK, et al. Amoebic liver abscess in Eastern Uttar Pradesh, India. Trop Geogr Med 1973 Sep;25(3):217-22.

4. Oschner A, DeBakey M, Murray S. Pyogenic abscesses of the liver, Am J Surg 1938;11:292-319.

5. Raghavan P, Kurein J, et al. Amoebic abscess epidemiology. J Ass Phys Ind 1961;9:568.

6. Habibullah CM, Afzal Qureshi M. Amobic liver abscess: A study. J Ass Phys Ind 1977;25:593.

7. Katzenstein D, Rickerson V, Braude A. New concepts of amebic liver abscess derived from hepatic imaging, serodiagnosis and hepatic enzymes in 67 consecutive cases in San Diego Medicine 1982;61:237-246.

8. Barbour GL and Juniper K Jr 1972. A clinical comparison of amoebic and pyogenic abscess of the liver in 66 patients. Am J Med 53:323-334.

9. Nanda RB, Tandon BN. A study of amoebic liver abscess. J Indian Med Assoc 1973 Aug 16;61(4):173-6.

10. Cruz I, Carneiro Chaves FJ. Hepatic amebiasis: experience in the therapy of 56 cases. Acta Med Port. 1981;(Suppl 2):103-10.

11. Poulose KP, Sahajanandan VK, Mohankumar VK, et al. Resolution of amoebic liver abscess, studied by serial liver scanning. J Assoc Physicians India. 1974 Mar;22(3):237-41.

12. Mehta AJ, Vakil BJ. A clinical study of 158 cases of amoebic liver abscess. Indian J Med Sci 1970 Aug;24(8):478-83.

13. Joshi VR, Kapoor OP, et al. J Ass Phys Ind 1972;20:761.

14. Manson-Bahr PEC and Apted FIC: Amoebiasis, Giardiasis and Balantidiasis. In "Manson's Tropical Diseases" Chapter 9, 18th Edition, Bailliere Tindall and ELBS London, 1982, pp. 121-145.

15. Adams EB and MacLeod IN: Invasive Amoebiasis. 1. Amoebic liver abscess and its complications. Medicine (Baltimore) 325-334, 1977. 